\title{
ISOMORPHIC GROUP RINGS WITH NONISOMORPHIC COMMUTATIVE COEFFICIENTS
}

\author{
JAN KREMPA
}

\begin{abstract}
Let $X$ be an infinite cyclic group. An example of two noncommutative nonisomorphic rings $R, S$ such that their group rings $R X, S X$ are isomorphic has been given in [1]. In the present note, we show that there also exist commutative nonisomorphic noetherian domains $A, B$ of Krull dimension 2 such that the group rings $A X, B X$ are isomorphic. That solves Problem 27 of [4] in the negative.
\end{abstract}

In what follows, some elementary properties of Dedekind domains contained in quadratic extensions of the field of rationals $Q$ will be used (cf. [2]). Let

$$
a=\frac{1+i \sqrt{47}}{2}, \quad D=Z[a], \quad K=Q(a) .
$$

If $\varphi$ is the nontrivial automorphism of $K$ then $\varphi$ is the only nontrivial automorphism of Dedekind domain $D$ and it induces an automorphism of the group of fractional ideals of $K$. Let $I$ be the ideal in $D$ generated by 2 and $a$. Then, it may be easily checked that $I$ is not a principal ideal, $I \cap \varphi(I)=2 D$ and $I^{5}=b D$, where $b=4+a$. Thus, if $\equiv$ means coincidence of classes of fractional ideals, then $\varphi(I) \equiv I^{4} \equiv I^{-1}$ and $\varphi\left(I^{-1}\right) \equiv I$. Hence, $I^{2}$ is not equivalent to $\varphi^{m}\left(I^{l}\right)$ where $m=1,2 ; l= \pm 1$. Now, let

$$
A=\bigoplus_{n=-\infty}^{\infty} I^{2 n} t^{n}, \quad B=\bigoplus_{n=-\infty}^{\infty} I^{n} t^{n} \subset K\langle t\rangle
$$

where $\langle t\rangle$ is an infinite cyclic group which is generated by $t$. We shall show that the rings $A, B$ are not isomorphic. Let us suppose $\sigma: A \rightarrow B$ is an isomorphism. Under localization at $S=Z \backslash\{0\}, \sigma$ becomes an isomorphism of $K\langle t\rangle$.

Hence $\sigma(K)=K$ and so $\sigma_{\mid K}=\varphi^{m}$, where $m=1$ or $m=2$ (cf. [4]). Moreover, $\sigma(t)=c t^{l}, c \in K^{*}, l= \pm 1$. Since $I^{l} t^{l}=\sigma\left(I^{2} t\right)=\sigma\left(I^{2}\right) c t^{l}$ we have $\sigma\left(I^{2}\right) \equiv I^{l}$. Thus $\varphi^{m}\left(I^{2}\right) \equiv I^{l}$, which is impossible.

Now, we shall establish an isomorphism of the group rings $A X, B X$. Let $\tau$ be the $K$-automorphism of $K\langle t\rangle$ given by formulas $\tau(t)=t^{2} x, \tau(x)=b t^{5} x^{2}$ where $x$ generates the group $X$. Then $\tau^{-1}$ is given by $\tau^{-1}(t)=b^{-1} t^{-2} x, \tau^{-1}(x)=b^{2} t^{5} x^{-2}$. Since $b$ generates $I^{5}$ then it may be computed that $\tau(A X) \subset B X$ and $\tau^{-1}(B X) \subset A X$. Thus, $\tau$ induces an isomorphism of the rings $A X, B X$. Similar examples may be constructed starting from some other Dedekind domains.

It is easily seen that the above-considered rings $A, B$ are finitely generated and hence noetherian. Moreover, the Krull dimension of $A, B$ equal to 2 . It is known 
that if $R, S$ are rings (not necessarily commutative) with classical Krull dimension 0 and $R X \simeq S X$ then $R \simeq S$. Moreover, there exist noncommutative and nonisomorphic noetherian domains $R, S$ of Krull dimension 1 such that $R X \simeq S X$, [3].

ADDED IN THE PROOF. Recently, a paper On the coefficient ring of a torus extension, Osaka J. Math. 17 (1980), 769-782, by K. Yoshida has appeared. Two-dimensional, nonisomorphic affine algebras, torus extensions of which are isomorphic, are constructed.

\section{REFERENCES}

1. L. Grunenfelder and M. M. Parmenter, Isomorphic group rings with nonisomorphic coefficient rings, Canad. Math. Bull. 23 (1980), 245-246.

2. H. Hasse, Number theory, Springer-Verlag, Berlin, 1979.

3. J. Krempa, Isomorphic group rings of free abelian groups, Canad. J. Math. (to appear).

4. S. K. Sehgal, Topics in group rings, Marcel Dekker, New York, 1978.

InstTtute of Mathematics, Universtty OF Warsaw, 00-901 Warsaw, Poland 